\title{
RESULTADOS DEL TRATAMIENTO QUIRÚRGICO DEL COLANGIOCARCINOMA INTRAHEPÁTICO*
}

\author{
Dr. Alexandre Sauré ${ }^{1}$ \\ ${ }^{1}$ Departamento de Cirugía, Hospital Clínico Universidad de Chile. \\ Santiago Chile.
}

\author{
TRABAJO DE INGRESO
}

\begin{abstract}
\section{Intrahepatic cholangiocarcinoma. Experience in 30 operated patients}

Background: Intrahepatic cholangiocarcinoma (ICC) corresponds to $10 \%$ of liver primary malignant tumors. Aim: To show the results of surgical treatment of ICC in a biliary surgery center. Material and Methods: Review of a prospective database of operated patients at a surgical service of a clinical hospital. Thirty operated patients with an ICC, aged 25 to 83 years $(20$ women), were identified. Results: Twenty six patients had symptoms, 12 of 19 had high levels of CA19-9 and in four the tumor was non resectable. Twenty four patients underwent major hepatectomy and two, a minor hepatectomy. Combined resections were performed in three patients. Lymphadenectomy was performed in 14 patients and five had lymph node metastases. Surgical time was 272 minutes, mean intensive care unit stay was 10 days and mean ventilatory support use was five days. Surgical mortality was $19 \%$ and complications appeared in $53 \%$ of patients. Tumors were stage I, II, III and IV in 11, 5, 3 and 11 patients respectively. Overall survival was 16 months. Survival in tumors stage I and II was 50\% at five years. In stages III and IV, it was 11.2 months. Conclusions: Surgery for ICC has an acceptable mortality and complications rate with a five years survival of $25 \%$.
\end{abstract}

Key words: Intrahepatic cholangiocarcinoma, survival, hepatectomy.

\section{Resumen}

Introducción: El colangiocarcinoma intrahepático $(\mathrm{CCIH})$, corresponde al segundo cáncer primario hepático, representando alrededor del 10\% de los cánceres primarios del hígado; el tratamiento es la hepatectomía. Objetivo: Mostrar los resultados del tratamiento quirúrgico de pacientes con $\mathrm{CCIH}$ en Chile en un centro de cirugía hepatobiliar. Pacientes y Métodos: Análisis prospectivo de los pacientes con CCIH operados en nuestro centro entre 2005 y 2015. Resultados: 30 pacientes, 20 mujeres (67\%), 10 hombres (33\%). Edad promedio: 60 años (rango 25-83 ), 26 pacientes sintomáticos (87\%), CA19-9 elevado en 12 $(63,2 \%), 4$ pacientes $(13 \%)$ irresecables, 24 pacientes $(80 \%)$ hepatectomías mayores y 2 resecciones menores. Resecciones combinadas en 3, linfadenectomía en 14 pacientes (47\%), metástasis linfonodales en 5 (17\%).

*Recibido el 11 de junio de 2015 y aceptado para publicación el 21 de julio de 2015.

El autor no refiere conflictos de interés.

Correspondencia: Dr. Alexandre Sauré M. asaurem@gmail.com 
Tiempo promedio quirúrgico 272 min (rango 45-480). UCI, mediana 10 días, ventilación mecánica mediana 5 días. Hospitalización, mediana 10 días y 7 días postoperatorio. Morbilidad 53\%, mortalidad 19\%. R0 en 19 pacientes $(64 \%)$, R1 en 7 pacientes (23\%) y R2 en 4 pacientes (13\%). Estadio I en 11 pacientes, estadio II en 5 pacientes, estadio III en 3 pacientes, estadio IV en 11 pacientes. Sobrevida general de 16 meses. Sobrevida estadio I 58,4 meses, estadio II 31,1 meses, estadio III 5,9 meses y estadio IV 11,8 meses, $p=0,06$. Sobrevida R0 16 meses, R1: 31,1 meses y R2: 9,25 meses, $p=0,53$. Sobrevida estadios I y II $50 \%$ a 5 años, estadios IIIIV 11,2 meses, $\mathrm{p}<0,01$. Discusión: Este es el primer reporte nacional de una serie importante de pacientes con CCIH, con morbilidad y mortalidad aceptables. Sobrevida de $25 \%$ a 5 años.

Palabras clave: Hígado, colangiocarcinoma intrahepático, hepatectomía.

\section{Introducción}

El colangiocarcinoma intrahepático $(\mathrm{CCIH})$, es un adenocarcinoma primario del hígado que probablemente se origina de epitelio biliar periférico. Corresponde al segundo cáncer primario hepático luego del hepatocarcinoma; representando cerca del $10 \%$ de los cánceres primarios del hígado, con una incidencia mundial en aumento ${ }^{1-4}$. Se conocen algunos factores de riesgo como las infecciones parasitarias, la colangitis esclerosante primaria, anormalidades anatómicas, hepatolitiasis e infecciones virales, los cuales se logran determinar en la minoría de los pacientes ${ }^{5-9}$. Su presentación clínica tiende a ser más bien asintomática, por lo cual al momento del diagnóstico, muchas veces ya existen metástasis intrahepáticas, metástasis ganglionares o invasión vascular y por lo tanto, la resecabilidad se logra solamente entre un $19 \%-74 \%$ de los casos $^{10-12}$. Si a esto le agregamos el hecho que el mejor tratamiento hasta el momento para estos pacientes es la cirugía radical $\mathrm{R} 0$, se entiende por qué la sobrevida a 5 años es de $13-40 \%$ con una mediana de $18-37$ meses $^{13-17}$.

La mayoría de las series publicadas acerca de este tema abarcan pocos pacientes a lo largo de muchos años, lo que genera diferencias significativas con respecto al manejo ${ }^{18,19}$.

El objetivo del presente estudio es mostrar los resultados del tratamiento quirúrgico de pacientes con $\mathrm{CCIH}$ en un centro de cirugía hepatobiliar en Chile, ya que no existen publicaciones previas sobre este tópico en nuestro país: Se presentan los resultados referentes a morbimortalidad postoperatoria, tasa de resecabilidad y sobrevida a largo plazo.

\section{Pacientes y Métodos}

\section{Pacientes estudiados}

Se realizó una búsqueda en la base de datos prospectiva de Oncología del Departamento de Cirugía del Hospital Clínico de la Universidad de Chile, creada el 2004, para pacientes cuyos resultados histológicos fueran de $\mathrm{CCIH}$. Por lo tanto, corresponde a una serie de casos consecutivos con seguimiento prospectivo. Se excluyeron pacientes con colangiocarcinoma de la bifurcación de los hepáticos y colangiocarcinoma de colédoco distal. Entre enero de 2005 y diciembre de 2013 se operaron 30 pacientes con este diagnóstico. Para el análisis de sobrevida se incluyeron sólo los pacientes con seguimiento mayor a 1 año, por lo que se excluyeron 2 pacientes con menos de un año de seguimiento y 5 pacientes que fallecieron en el postoperatorio inmediato.

En el estudio preoperatorio se analizaron numerosas variables en todos los pacientes: características clínicas (género, edad, síntomas), marcadores tumorales como el CA19-9, antígeno carcinoembrionario y alfafetoproteína, exámenes de laboratorio (hemograma, perfil hepático, marcadores de la hepatitis, imágenes como ecotomografía abdominal, tomografía computada y/o resonancia magnética).

\section{Procedimiento quirúrgico}

Durante la cirugía se realizó de forma rutinaria ecografía intraoperatoria, para evaluar presencia de metástasis intrahepática y la relación del tumor con los vasos sanguíneos. La extensión de la resección se definió de acuerdo a la clasificación de Brisbane ${ }^{20}$. Se definió mortalidad quirúrgica como el fallecimiento ocurrido hasta 1 mes después de la cirugía Los criterios de irresecabilidad correspondieron a la presencia de metástasis a distancia, carcinomatosis peritoneal y/o compromiso vascular extenso. El grado de resección se definió de la siguiente forma: $\mathrm{R} 0$ cuando el margen quirúrgico es negativo en la biopsia definitiva, R1 cuando el margen quirúrgico es positivo en la biopsia definitiva y R2 cuando se apreció tumor residual intraoperatorio. Se realizó la etapificación tumoral de acuerdo a la $7^{\mathrm{a}}$ edición del American Joint Committee on Cancer (AJCC).

\section{Estudio anatomopatológico}

La apariencia del tumor al corte se clasificó en 3 tipos: nodular, intraductal e infiltrativo, de acuerdo a la clasificación propuesta por el grupo de estudio de cáncer hepático de Japón ${ }^{21}$. Todas las piezas fueron enviadas a anatomía patológica donde se determinó 
el tipo histológico, tamaño, número de lesiones, presencia de metástasis linfonodos y la presencia o ausencia de infiltración tumoral en el borde quirúrgico.

\section{Complicaciones postoperatorias}

Para la clasificación de la severidad de las complicaciones y la mortalidad postoperatoria hasta los 30 días, se empleó la propuesta por Clavien et $\mathrm{al}^{22,23}$, que los categoriza de grado I al V. Esta última corresponde a la mortalidad operatoria. Esta clasificación es la usada en forma internacional para evaluar la evolución postoperatoria y ha sido demostrada que es fácilmente reproducible y objetiva.

\section{Seguimiento}

Al alta los pacientes se controlaron en forma regular con exámenes generales de laboratorio, marcadores tumorales e imágenes (TC o RNM) cada 3 meses el primer año, cada 6 meses el segundo año y anualmente hasta completar 5 años de seguimiento. Se actualizó la sobrevida de acuerdo a informes del Registro Civil.

\section{Análisis estadístico}

La variables continuas se expresaron como promedios y desviación standard. Las variables categóricas se presentaron como frecuencia y porcentajes.

Para el análisis de sobrevida se utilizó el método de Kaplan-Meier. Para el análisis de factores independientes asociados a la sobrevida acumulada se utilizó el análisis de regresión proporcional de Cox. Un valor de $\mathrm{p}<0,05$ se consideró estadísticamente significativo.

\section{Aspectos éticos}

La información se manejó sólo por el autor, en forma anónima. No existe ningún conflicto de interés en este trabajo.

\section{Resultados}

Hubo un total de 30 pacientes, 20 mujeres $(66,6 \%)$ y 10 hombres, con una edad promedio de 60 años (rango entre los 25 y 83 años). Del grupo total, 26 pacientes tuvieron manifestaciones clínicas en el preoperatorio. El síntoma más frecuente fue dolor abdominal $(\mathrm{n}=17)$, seguido por baja de peso y anorexia $(n=7)$, lumbago $(n=2)$, hepatomegalia $(\mathrm{n}=3)$, fiebre $(\mathrm{n}=2)$, dolor óseo $(\mathrm{n}=1)$ e ictericia $(\mathrm{n}=2)$. El tumor se encontró de forma incidental por exámenes de imágenes en 5 pacientes $(17 \%)$ (Tabla 1).

Ningún paciente presentaba enfermedad hepática asociada con anticuerpos para hepatitis B y C que fueron negativos. Sin embargo, se observó cambios en el perfil hepático (Tabla 2) de tipo ictericia obstructiva.

El CA19-9 se determinó en 19 pacientes, estando con cifras elevadas en $12(63,2 \%)$ con un valor promedio de $150 \mathrm{u} / \mathrm{ml}$ (mediana) con un rango de 1 a $4.770 \mathrm{u} / \mathrm{ml}$, siendo el valor normal hasta $37 \mathrm{u} / \mathrm{ml}$.

En 6 pacientes se sospechó compromiso vascular por imágenes preoperatorias.

Tabla 1. Características generales de pacientes con CCIH. $n=30$

\begin{tabular}{|lcc|}
\hline Edad (años) & $\begin{array}{c}60 \pm 14,5 \\
(25-81)\end{array}$ & \\
\hline Género & $\begin{array}{c}\text { Femenino } 67 \% \\
\text { IMC }\left(\mathrm{kg} / \mathrm{m}^{2}\right)\end{array}$ & $\begin{array}{l}\text { Masculino } 33 \% \\
(21,4 \pm 5,8\end{array}$ \\
\hline Síntomas y signos & $\mathbf{n}$ & \\
\hline Dolor abdominal & 17 & $\%$ \\
\hline Baja de peso & 7 & 57 \\
Lumbago & 2 & 23 \\
\hline Fiebre & 2 & 6 \\
Dolor óseo & 1 & 3 \\
Hepatomegalia & 3 & 10 \\
Ictericia & 2 & 6 \\
\hline Asintomáticos & 4 & 13 \\
\hline
\end{tabular}

$\mathrm{IMC}=$ índice masa corporal. $\mathrm{CCIH}=$ colangiocarcinoma intrahepático.

Tabla 2. Exámenes de laboratorio en pacientes con CCIH. $n=30$

\begin{tabular}{|ll|}
\hline Parámetro & Promedio \pm DS \\
\hline Bilirrubina total $(\mathrm{mg} / \mathrm{dl})$ & $2,28 \pm 6,1$ \\
\hline Fosfatasas alcalina $(\mathrm{u} / \mathrm{lt})$ & $184 \pm 176$ \\
\hline Gama glutamil transpeptidasa $(\mathrm{u} / \mathrm{lt})$ & $146 \pm 156$ \\
Transaminas oxaloacética $(\mathrm{u} / \mathrm{lt})$ & $47,8 \pm 47$ \\
\hline Transaminas pirúvica $(\mathrm{u} / \mathrm{lt})$ & $36,1 \pm 36$ \\
Glicemia $(\mathrm{mg} / \mathrm{dl})$ & $118 \pm 47$ \\
\hline Tiempo protrombina $(\%)$ & $92,7 \pm 31$ \\
\hline Albúmina (g/dl) & $3,6 \pm 1$ \\
\hline Hematocrito $(\%)$ & $37 \pm 9,8$ \\
Hemoglobina (g/dl) & $15,2 \pm 8$ \\
Recuento plaquetas & $221.000 \pm 99.000$ \\
Creatininemia (mg/dl) & $0,9 \pm 0,8$ \\
\hline
\end{tabular}


Tabla 3. Procedimientos quirúrgicos

\begin{tabular}{|lccccc|}
\hline Tipo de cirugía & $\mathbf{n}$ & $\mathbf{\%}$ & Tiempo Op. (min) & Ro & Mortalidad \\
\hline Trisegmentectomía derecha & 10 & 32 & 320 & 7 & $4(40 \%)$ \\
\hline Trisegmentectomía izquierda & 1 & 3,5 & 270 & 1 & \\
Bisegmentectomía derecha & 6 & 19 & 274 & 3 & $1(25 \%)$ \\
Bisegmentectomía izquierda & 4 & 13 & 289 & 3 & 5 \\
Segmentectomía & 5 & 16 & 443 & 0 & - \\
Biopsia & 4 & 13 & 94 & - & \\
\hline Ligadura portal & 1 & 3,5 & 90 & & \\
\hline
\end{tabular}

Mediana del total pctes: 274 (64-443); Mediana de resecciones: 289 (270-443); Promedio del grupo: 250.

En 3 pacientes se realizó biopsia preoperatoria dando como resultado en 2 pacientes colangiocarcinoma y en 1 adenocarcinoma mal diferenciado.

En 4 pacientes (13\%) no se realizó cirugía resectiva, sino sólo biopsia incisional que presentó un tumor irresecable con compromiso vascular y metástasis a distancia.

En 26 pacientes (87\%) se realizaron hepatectomías con intención curativa: 24 pacientes $(80 \%)$ fueron sometidos a hepatectomías mayores (tres o más segmentos de Couinaud), y 2 pacientes se sometieron a resecciones menores (Tabla 3 ). En esta misma tabla se exponen los pacientes que quedaron R0, el tiempo promedio operatorio y la mortalidad operatoria. Se aprecia que la trisegmentectomía hepática tuvo una mortalidad de $40 \%$ y la bisegmentectomía izquierda una mortalidad de $25 \%$.

Se efectuaron resecciones combinadas en 3 pacientes: en 1 paciente se resecó en forma parcial el diafragma derecho, en 1 paciente se resecó la vía biliar principal y en 1 paciente se resecó la vena porta con interposición de prótesis de PTFE anillada.

Se realizó linfadenectomía en 14 pacientes (47\%); encontrándose metástasis linfonodales en 5 pacientes $(17 \%)$.

El tiempo promedio quirúrgico fue de $272 \mathrm{~min}$ (rango 45-480 $\mathrm{min}$ ). Cinco pacientes (17\%) requirieron de transfusión intraoperatoria.

Los pacientes que requirieron UCI estuvieron una mediana de 10 días y con 0 ventilación mecánica una mediana de 5 días. Por otro lado estuvieron hospitalizados como mediana de 10 días en total y 7 días postoperatorio (Tabla 4).

Las complicaciones postoperatorias y la mortalidad se señalan en la Tabla 5. Hubo 2 complicaciones grado II $(7,7 \%)$ y 11 grado IIIa $(42,3 \%)$. La complicación más frecuente fue la fístula biliar seguida de colección abdominal.

La mortalidad (grado $\mathrm{V}$ de la clasificación) se observó en 5 pacientes $(19,2 \%)$ por insuficiencia hepática, shock postoperatorio y hepatitis isquémica por trombosis porta.

En 22 pacientes se identificaron lesiones únicas (73\%) con un promedio de diámetro máximo de 7,9 cm (rango 2,5-20 cm) (Tabla 6).

Para el tipo de resección se logró R0 en 18 pacientes $(60 \%), \mathrm{R} 1$ en 7 pacientes $(23 \%)$ y R 2 en 5 pacientes (17\%) (Tabla 7).

El tipo macroscópico de crecimiento tumoral fue: 26 pacientes $(87 \%)$ tipo nodular, 1 paciente $(3 \%)$ tipo periductal y 2 pacientes $(10 \%)$ tipo infiltrativo (Tabla 7).

Tabla 4. Tiempo quirúrgico y postoperatorio. $n=30$

\begin{tabular}{lr} 
1. Duración operación $(\min )$ & $272 \pm 117$ \\
2. Estadía postoperatoria (días) & $10[3-69]$ \\
UCI & $10[1-12]$ \\
Ventilación mecánica & $5[1-12]$ \\
Estada hospitalaria total & $7[1-68]$ \\
\hline
\end{tabular}

Tabla 5.

\begin{tabular}{|llcc|}
\hline & & n & \% \\
I & Fístula biliar & 3 & 10 \\
IIIa & Colección ascítica & 2 & 23 \\
& Fístula biliar (colección) & 4 & \\
& Hematoma & 1 & \\
IIIb & Estenosis CHD & 1 & 3 \\
V & Insuficiencia hepática & 2 & 17 \\
& Shock postoperatorio & 2 & \\
& Hepatitis isquemia & 1 & \\
\hline
\end{tabular}

*16 complicaciones en 16 pacientes. 53\% morbilidad. 
Tabla 6. Mortalidad operatoria

\begin{tabular}{|lll|}
\hline Mortalidad total & $30 / 5$ & $17 \%$ \\
\hline Mortalidad sin laparotomía exploradora & $26 / 5$ & $19,20 \%$ \\
\hline $\begin{array}{ll}\text { Mortalidad equipo HPB } \\
\text { Mortalidad equipo HPB sin resección }\end{array}$ & $23 / 3$ & $13 \%$ \\
\begin{tabular}{l} 
vascular \\
\hline
\end{tabular} & $4,76 \%$ \\
\hline
\end{tabular}

Del grupo sólo 2 pacientes (8\%) recibieron quimioterapia adyuvante.

La clasificación $T$ de acuerdo a la $7^{\text {a }}$ edición de la AJCC/UICC fue la siguiente: T1, 13 (43\%); T2, 8 (27\%); T3, 3 (10\%); T4, 2 (7\%); y Tx, 4 (13\%). La clasificación N fue la siguiente: N0, $8(27 \%)$; N1, $5(17 \%)$ y Nx, $15(50 \%)$. La clasificación $\mathrm{M}$ fue la siguiente: M0, 25 (83\%) y M1, 5 (17\%).

De acuerdo a la $7^{\mathrm{a}}$ edición de la AJJCC/UICC la etapificación fue: Estadio I: 11 pacientes, estadio II: 5 pacientes, estadio III: 3 pacientes, estadio IV: 11 pacientes.

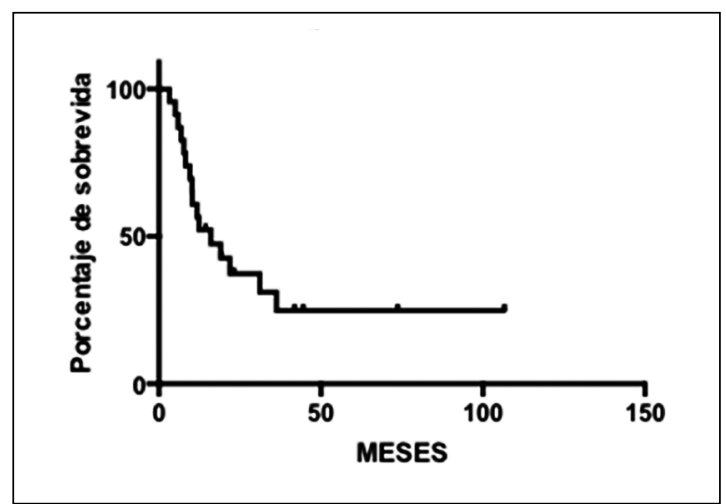

Figura 1. Sobrevida general.

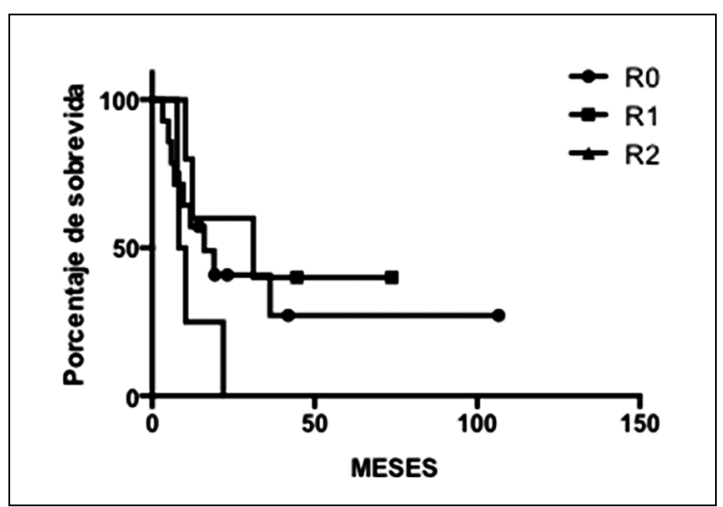

Figura 3. Sobrevida por resección.
La sobrevida general del grupo fue de 16 meses (Figura 1). Si se separa la sobrevida por estadio se obtiene que en el estadio I, la sobrevida es de 58,4 meses, en el estadio II de 31,1 meses, en el estadio III de 5,9 meses y en el estadio IV de 11,8 meses con un valor $p=0,06$ (Figura 2). Si se separa por márgenes quirúrgicos se obtiene que la sobrevida en $\mathrm{R} 0$ es de 16 meses, en R 1 de 31,1 meses y en R 2 de 9,25 meses con un valor $p=0,53$ (Figura 3 ).

Si se evalúa la sobrevida separando por estadios precoces contra tardíos se obtiene que en estadios I y II se logra un $50 \%$ de sobrevida a 5 años y los estadios III-IV tienen una sobrevida de 11,2 meses con un valor $\mathrm{p}<0,01$ (Figura 4).

Si se evalúa sobrevida separando lesiones únicas contra múltiples se observa que en lesiones únicas la sobrevida es de 41,9 meses y para las múltiples 21,9 meses con un valor $\mathrm{p}=0,8$ (Tabla 8 ).

También se analizaron como variables independientes para la sobrevida: el tamaño tumoral y el número de ganglios metastásicos, sin encontrar diferencias significativas.

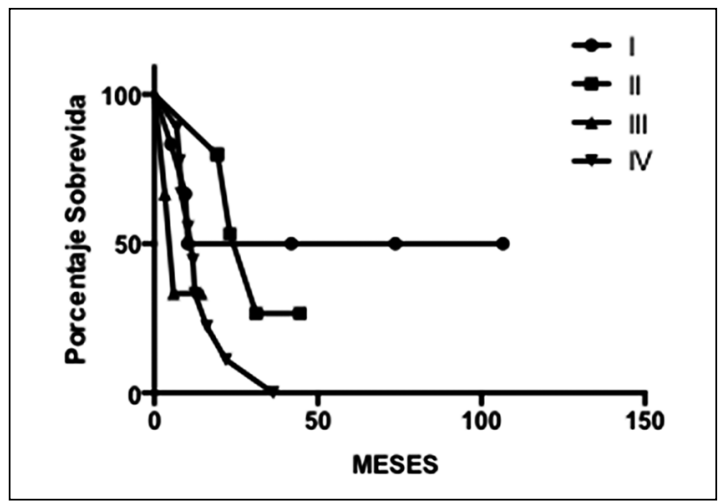

Figura 2. Sobrevida por estadio.

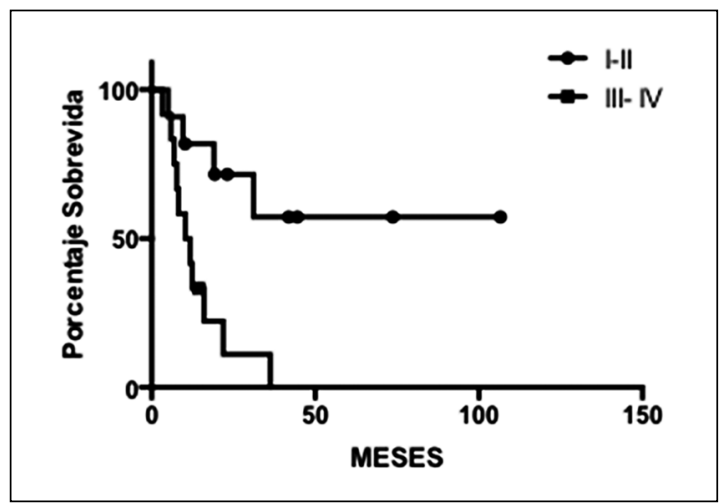

Figura 4. Sobrevida por resección precoz vs tardía. 
Tabla 7. Características del tumor

\begin{tabular}{|lc|}
\hline Tamaño (cm) & $\mathbf{8 , 2} \pm \mathbf{4 , 2}(\mathbf{3 - 2 0})$ \\
\hline Tipo macroscópico & \\
a. Nodular & 26 \\
b. Periductal & 2 \\
c. Infiltrativa & 3 \\
\hline
\end{tabular}

\section{Discusión}

Históricamente el colangiocarcinoma intrahepático se reconocía como un raro tumor de las vías biliares. De hecho, hasta la $6^{\mathrm{a}}$ clasificación de la AJCC se etapificaba junto al hepatocarcinoma. Sin embargo, en los últimos años se ha podido apreciar un aumento en su incidencia. Una posible explicación para

Tabla 8. Características anatomopatológicas y sobrevida

\begin{tabular}{|c|c|c|c|c|c|c|}
\hline \multirow[b]{2}{*}{ Variable } & \multirow[b]{2}{*}{$\mathbf{n}$} & & \multicolumn{2}{|c|}{ Sobrevida \% } & \multirow[b]{2}{*}{5 años } & \multirow[b]{2}{*}{$\mathbf{p}$} \\
\hline & & $\%$ & 1 año & 3 años & & \\
\hline \multicolumn{7}{|c|}{ 1. Márgenes resección } \\
\hline R0 & 19 & 63 & 53 & 23 & 23 & $>0,5$ \\
\hline R1 & 7 & 23 & 60 & 40 & 40 & \\
\hline R2 & 4 & 13 & 40 & 0 & 0 & \\
\hline \multicolumn{7}{|l|}{ 2. $\mathrm{T}$} \\
\hline $\mathrm{T} 1$ & 13 & 43 & 37 & 37 & 37 & $>0,39$ \\
\hline $\mathrm{T} 2$ & 8 & 27 & 85 & 23 & 0 & \\
\hline $\mathrm{T} 3$ & 3 & 10 & 33 & 0 & 0 & \\
\hline $\mathrm{T} 4$ & 2 & 7 & 0 & 0 & 0 & \\
\hline \multicolumn{7}{|c|}{ 3. Estadio UICC } \\
\hline I y II & 16 & 53 & 72 & 50 & 50 & $<0,01$ \\
\hline III y IV & 14 & 47 & 33 & 0 & 0 & \\
\hline \multicolumn{7}{|c|}{ 4. Número tumor } \\
\hline Único & 22 & 73 & 69 & 20 & 20 & $>0,46$ \\
\hline Múltiples & 8 & 27 & 33 & 33 & 33 & \\
\hline
\end{tabular}

Tabla 9. Resumen de resultados de autores extranjeros

\begin{tabular}{|lccccc|}
\hline Autor & Año & n & Resecados (\%) & Sobrevida 3 años & Sobrevida 5 años \\
Harrison et al & 1998 & 32 & - & - & 42 \\
Weimann et al & 2000 & 162 & 59 & 31 & 21 \\
Inoue et al & 2000 & 52 & - & - & 36 \\
Weber et al & 2001 & 53 & 62 & 22 & - \\
Jan et al & 2004 & 373 & 36 & 9,2 & 4,1 \\
Lang et al & 2005 & 50 & 54 & 82 & - \\
Paik et al & 2007 & 97 & - & 52 & 31 \\
De Oliveira et al & 2007 & 44 & 66 & - & 63 \\
Nathan et al & 2007 & 591 & - & - & 17 \\
Konstadoulakis & 2007 & 72 & 71 & 49 & 25 \\
Nakagohri et al & 2008 & 56 & - & - & 32 \\
Tamandl et al & 2008 & 74 & - & 45 & 28 \\
Endo et al & 2008 & 77 & 32 & - & - \\
Sauré & 2015 & 30 & 83 & 25 & 25 \\
\hline
\end{tabular}


esto sería el aumento en la incidencia de virus hepatitis $\mathrm{C}$ y en un mejor sistema de clasificación ${ }^{23-31}$.

Si bien se describen una importante lista de factores de riesgo, la mayoría de los casos se presenta en pacientes sin antecedentes de importancia, con síntomas inespecíficos y en estadios avanzados ${ }^{32-36}$. Esto explica que su resecabilidad sea baja y que los pacientes que sí llegan a resecarse, sean sometidos a resecciones hepáticas mayores, asociándose, en muchos casos con resecciones extendidas a órganos vecinos.

Por lo anterior, la sobrevida a 5 años publicada varía entre 4,1 y $43,7 \%$, lo cual se asemeja a los resultados de este estudio en donde la sobrevida a 5 años del grupo fue de $25 \%$ (Tabla 8 ). Se sabe que el mejor tratamiento para estos pacientes es la resección R0, pero esto no siempre es posible, identificándose enfermedad residual hasta en un $40 \%$ de los pacientes. Sin embargo, los pacientes con resecciones R1 han demostrado tener mejor sobrevida que los pacientes irresecables (R2). Más del $80 \%$ de las cirugías realizadas en nuestro centro fueron hepatectomías mayores, lo cual a su vez se acompañó de una morbilidad del 50\% y una mortalidad postoperatoria del $19 \%$, lo cual es semejante con experiencias internacionales ${ }^{1,35}$. Si bien en nuestros pacientes observamos una mejor sobrevida en los pacientes R0 y R1 esta no fue estadísticamente significativa comparada con los pacientes R2. Sin embargo, al comparar los grupos con enfermedad precoz (estadios I-II) y tardía (III-IV) se logra observar una significativa mayor sobrevida en los pacientes con enfermedad inicial.

Las características que han demostrado influir negativamente en la sobrevida de los pacientes con esta enfermedad son: lesiones intrahepáticas múltiples, infiltración vascular, metástasis linfonodales y tumor del subtipo infiltrante periductal ${ }^{15,16,38-41}$.

Otras características que sólo algunos estudios han asociado a peor pronóstico se refieren a: los pacientes jóvenes y niveles de CA19-9 elevados al momento del diagnóstico. En este estudio ninguna de estas variables logró significación estadística ${ }^{10,39,42}$.

Con respecto al uso de otras terapias, se han probado múltiples alternativas: quimioterapia, radioterapia, radiofrecuencia y quimioembolización transarterial ${ }^{1,38}$. Lamentablemente la mayoría de estos estudios tienen una muestra escasa, son estudios retrospectivos y su principal rol estaría en pacientes irresecables. Según Valle et al, la quimioterapia adyuvante lograría un aumento de 3 meses en la sobrevida ${ }^{43}$.

Este es el primer reporte nacional de una serie importante de pacientes con colangiocarcinoma intrahepático (Tabla 9). En base a nuestros hallazgos y a lo publicado en el mundo, es posible concluir que los centros hepatobiliares chilenos se enfrentarán con más frecuencia a este agresivo tumor, el cual se diagnostica muchas veces en estadios avanzados, requiriendo para su óptimo manejo, de grandes resecciones hepáticas.

\section{Referencias}

1. Brown KM, Parmar AD, Geller DA. Intrahepatic cholangiocarcinoma. Surg Oncol Clin N Am. 2014;23:23146.

2. Khan SA, Davidson BR, Goldin R, Pereira SP, Rosenberg WM, Taylor-Robinson SD, et al. Guidelines for the diagnosis and treat-ment of cholangiocarcinoma: consensus document. Gut 2002;51(Suppl 6): VI1-9.

3. Khan SA, Toledano MB, Taylor-Robinson SD. Epidemiology, risk factors, and pathogenesis of cholangiocarcinoma. HPB (Oxford) 2008;10:77-82.

4. Shaib Y, El-Serag HB. The epidemiology of cholangiocarcinoma. Semin Liver Dis. 2004;24:115-25.

5. Dodson RM, Weiss MJ, Cosgrove D, Herman JM, Kamel I, Anders R, et al. Intrahepatic cholangiocarcinoma: management options and emerging therapies. J Am Coll Surg. 2013;217:736-50.

6. Palmer WC, Patel T. Are common factors involved in the pathogenesis of primary liver cancers? A metaanalysis of risk factors for intrahepatic cholangiocarcinoma. J Hepatol. 2012;57:69-76.

7. Hammill CW, Wong LL. Intrahepatic cholangiocarcinoma: a malignancy of increasing importance. J Am Coll Surg. 2008;207:594-603.

8. Shaib YH, El-Serag HB, Davila JA, Morgan R, McGlynn KA. Risk factors of intrahepatic cholangiocarcinoma in the United States: a case-control study. Gastroenterology 2005;128:620-6.

9. Patel T. Increasing incidence and mortality of primary intrahepatic cholangiocar- cinoma in the United States. Hepatology 2001;33:1353-7.

10. Guglielmi A, Ruzzenente A, Campagnaro T, Pachera S, Valdegamberi A, Nicoli P, et al. Intrahepatic cholangiocarcinoma: prognostic factors after surgical resection. World J Surg. 2009;33:1247-54.

11. Puhalla H, Schuell B, Pokorny H, Kornek GV, Scheithauer W, Gruenberger T. Treatment and outcome of intrahepatic cholangiocellular carcinoma. Am J Surg. 2005;189:173-7.

12. Nakagohri T, Asano T, Kinoshita H, Kenmochi $T$, Urashima T, Miura F, et al. Aggressive surgical resection for hilar-invasive and peripheral intrahepatic cholangiocarcinoma. World J Surg. 2003;27:289-93.

13. Chen LP, Li C, Wang C, Wen TF, Yan LN, Li B. Predictive factors of recurrence for patients with intrahepatic cholangiocarcinoma after hepatectomy. Hepatogastroenterology 2012;59:1765-8.

14. Farges O, Fuks D. Clinical presentation and manage- 
ment of intrahepatic cholangiocarcinoma. Gastroenterol Clin Biol. 2010;34:191-9.

15. Cho SY, Park SJ, Kim SH, Han SS, Kim YK, Lee KW, et al. Survival analysis of intrahepatic cholangiocarcinoma after resection. Ann Surg Oncol. 2010;17:182330 .

16. Shirabe K, Mano Y, Taketomi A, Soejima Y, Uchiyama $\mathrm{H}$, Aishima S, et al. Clinicopathological prognostic factors after hepatectomy for patients with massforming type intrahepatic cholangiocarcinoma: relevance of the lymphatic invasion index. Ann Surg Oncol. 2010;17:1816-22.

17. Nathan H, Aloia TA, Vauthey JN, Abdalla EK, Zhu AX, Schulick RD, et al. A proposed staging system for intrahepatic cholangiocarcinoma. Ann Surg Oncol. 2009;16:14-22.

18. Saiura A, Yamamoto J, Kokudo N, Koga R, Seki M, Hiki N, et al. Intrahepatic cholangiocarcinoma: analysis of 44 consecutive resected cases including 5 cases with repeat resections. Am J Surg. 2011;201:203-8.

19. Tamandl D, Herberger B, Gruenberger B, Puhalla H, Klinger M, Gruenberger T. Influence of hepatic resection margin on recurrence and survival in intrahepatic cholangiocarcinoma. Ann Surg Oncol. 2008;15:278794.

20. IHPBA Brisbane 2000 Terminology of Liver anatomy and resections. HPB 2000;2:333-9.

21. Clarien P, Sarabria J, Strasberg S. Proposed classification of complications of surgery with examples of utility in cholecystectomy. Surgery 1992;111:518-26.

22. Dindo D, Demartines N, Clarier PA. Classification of surgical complications. Ann Surg. 2004;240:205-13.

23. Zhou Y, Zhao Y, Li B, Huang J, Wu L, Xu D, et al. Hepatitis viruses infection and risk of intrahepatic cholangiocarcinoma: evidence from a meta-analysis. BMC Cancer 2012;12:289-97.

24. Nkontchou G, Tran Van Nhieu J, Ziol M, Tengher I, Mahmoudi A, Roulot D, et al. Peripheral intrahepatic cholangiocarcinoma occurring in patients without cirrhosis or chronic bile duct diseases: epidemiology and histopathology of distant nontumoral liver in 57 white patients. Eur J Gastroenterol Hepatol. 2013;25:94-8.

25. Sempoux C, Jibara G, Ward SC, Fan C, Qin L, Roayaie $\mathrm{S}$, et al. Intrahepatic cholangiocarcinoma: new insights in pathology. Semin Liver Dis. 2011;31:49-60.

26. Patel T. Worldwide trends in mortality from biliary tract malignancies. BMC Cancer 2002;2:10.

27. Yang J, Yan LN. Current status of intrahepatic cholangiocarcinoma. World J Gastroenterol. 2008;14:6289-97.

28. de Groen PC, Gores GJ, LaRusso NF, Gunderson LL, Nagorney DM. Biliary tract cancers. N Engl J Med. 1999;341:1368-78.

29. Dhanasekaran R, Hemming AW, Zendejas I, George T, Nelson DR, Soldevila-Pico C, et al. Treatment outcomes and prognostic factors of intrahepatic cholangiocarcinoma. Oncol Rep. 2013;29:1259-67.
30. Yamasaki S. Intrahepatic cholangiocarcinoma: macroscopic type and stage classification. J Hepatobiliary Pancreat Surg. 2003;10:288-91.

31. Edge SB, Compton CC. The American Joint Committee on Cancer: the 7th edition of the AJCC cancer staging manual and the future of TNM. Ann Surg Oncol. 2010;17:1471-4.

32. De Oliveira ML, Cunningham SC, Cameron JL, Kamangar F, Winter JM, Lillemoe KD, et al. Cholangiocarcinoma: thirty-one-year experience with 564 patients at a single institution. Ann Surg. 2007;245:755-62.

33. Paik KY, Jung JC, Heo JS, Choi SH, Choi DW, Kim YI. What prognostic factors are important for resected intrahepatic cholangiocarcinoma? J Gastroenterol Hepatol. 2008;23:766-70.

34. Endo I, Gonen M, Yopp AC, Dalal KM, Zhou Q, Klimstra $\mathrm{D}$, et al. Intrahepatic cholangiocarcinoma: rising frequency, improved survival, and determinants of outcome after resection. Ann Surg. 2008;248:84-96.

35. Blechacz B, Komuta M, Roskams T, Gores GJ. Clinical diagnosis and staging of cholangiocarcinoma. Nat Rev Gastroenterol Hepatol. 2011;8:512-22.

36. Shen WF, Zhong W, Xu F, Kan T, Geng L, Xie F, et al. Clinicopathological and prognostic analysis of 429 patients with intrahepatic cholangiocarcinoma. World J Gastroenterol. 2009;15:5976-82.

37. Yedibela S, Demir R, Zhang W, Meyer T, Hohenberger W, Schönleben F. Surgical treatment of mass-forming intrahepatic cholangiocarcinoma: an 11-year Western single-center experience in 107 patients. Ann Surg Oncol. 2009;16:404-12.

38. Carpizo DR, D’Angelica M. Management and extent of resection for intrahepatic cholangiocarcinoma. Surg Oncol Clin N Am. 2009;18:289-305.

39. Shimada K, Sano T, Sakamoto Y, Esaki M, Kosuge T, Ojima H. Surgical outcomes of the mass-forming plus periductal infiltrating types of intrahepatic cholangiocarcinoma: a comparative study with the typical massforming type of intrahepatic cholangiocarcinoma. World J Surg 2007;31:2016-22.

40. de Jong MC, Nathan H, Sotiropoulos GC, Paul A, Alexandrescu S, Marques H, et al. Intrahepatic cholangiocarcinoma: an international multi-institutional analysis of prognostic factors and lymph node assessment. J Clin Oncol. 2011;29:3140-5.

41. Suh KS, Roh HR, Koh YT, Lee KU, Park YH, Kim SW. Clinicopathologic features of the intraductal growth type of peripheral cholangiocarcinoma. Hepatology 2000;31:12-7.

42. Miller G, Schwartz LH, D'Angelica M. The use of imaging in the diagnosis and staging of hepatobiliary malignancies. Surg Oncol Clin N Am 2007;16:343-68.

43. Valle J, Wasan H, Palmer DH, Cunningham D, Anthoney A, Maraveyas A, et al. Cisplatin plus gemcitabine versus gemcitabine for biliary tract cancer. $\mathrm{N}$ Engl J Med. 2010;362:1273-81. 


\section{INFORME TRABAJO DE INGRESO}

Señor Presidente y miembros del Directorio de la Sociedad de Cirujanos de Chile:

En primer lugar quisiera agradecer a ustedes por haberme concedido el honor de comentar el trabajo de ingreso del Dr. Alexandre Sauré Maritano, que lleva el título de "Resultados del tratamiento quirúrgico del colangiocarcinoma intrahepático".

El colangiocarcinoma intrahepático (CCI) es tumor maligno derivado del epitelio de conductos biliares y corresponde a la segunda neoplasia maligna primaria más frecuente del hígado (10-20\%), aunque continúa siendo una patología relativamente rara, con una leve tendencia al aumento de su incidencia mundial. Es más frecuente sobre la sexta década de la vida, sin predominio por género. Se describen algunos factores asociados a su etiopatogenia, tales como: litiasis intrahepática, enfermedad de Caroli, quistes de colédoco, colangitis esclerosante, algunas parasitosis y enfermedad inflamatoria intestinal. $\mathrm{Su}$ presentación clínica suele ser inespecífica e insuficiente para establecer diagnóstico. En estadios precoces suele ser asintomática, y por el contrario, en estados más avanzados los pacientes se pueden presentar con compromiso del estado general, baja de peso, dolor abdominal, masa palpable y la menor de las veces ictericia $(<30 \%)$. En cuanto a pruebas de laboratorio, éstas suelen mostrar una alza en los niveles de fosfatasas alcalinas, GGT y CA 19-9. Sin duda, el principal método diagnóstico es el estudio de imágenes, y entre ellos cobra mayor importancia la tomografía axial computada (TAC) dinámica y la resonancia magnética, aunque en ocasiones no es posible diferenciar entre CCI de hepatocarcinoma celular, por lo cual, se hace necesario completar el estudio diagnóstico con histopatología. En cuanto a su terapia, la cirugía continúa siendo el principal y único tratamiento con intensión curativo, y en la mayor parte de las veces se requiere de hepatectomía mayores o extendidas $(70 \%)$, siendo aún discutible el rol de la linfadenectomía. Respecto al trasplante hepático, éste continúa siendo controversial, ya que la selección de pacientes y protocolos de terapias neoadyuvantes son muy dispares, además de presentar sobrevida (SV) a 3 años que no superan los resultados de la cirugía R0. Se describen como factores pronósticos el número de tumores, la invasión vascular, el compromiso nodal, el estadio según TNM (basado en la $7^{\mathrm{a}}$ edición de la AJCC) y la resección R0; otros factores, tales como marcadores tumorales, tamaño tumoral o características histopatológicas, son más controversiales, ya que en estudios multivariados no han logrado demostrar tener impacto en la SV de los pacientes.
El trabajo que el Dr. Sauré nos presenta, muestra la experiencia de un centro nacional, con larga trayectoria en la cirugía hepatobiliar. Este estudio, corresponde a un estudio de cohorte histórica, sobre una población de 30 pacientes reclutados de manera consecutiva en un período de 8 años. Es importante destacar, que hasta la fecha no existen otros reportes chilenos con esta expriencia y a nivel mundial los estudios más grandes corresponden a series de estudios multicéntricos y reclutados por largos períodos de tiempo. En el $80 \%$ de los pacientes se realizó hepatectomía mayor, con una tasa de mortalidad global del $19 \%$, siendo la mayoría de las veces asociada a estas últimas y quizás, por este motivo, además de una muestra insuficiente, se pueda explicar por qué la SV a 3 años es mayor en resecciones R1 y no en R0. También se debe destacar, que en esta serie se efectuó linfadenectomía en sólo un $47 \%$ de los pacientes resecados, y en el $35 \%$ de ellos se demostró metástasis ganglionar, lo cual coincide con otros reportes que describen metástasis ganglionares en un $30-36 \%$ de pacientes con linfadenectomía. Esto último tiene impacto en los análisis de SV, ya que en más del $50 \%$ de los pacientes resecados se desconoce el real estado nodal y por lo tanto, en la actualidad, se estima que la linfadenectomía permitiría disminuir la recurrencia local, y por sobretodo reetapificar oncológicamente al paciente. Otro factor pronóstico evaluado fue el número de tumores, y si bien sólo el $26 \%$ presentó más de una lesión tumoral, en estos pacientes la SV fue menor que en aquellos con lesión única, pero sin alcanzar significación estadística. Finalmente, sólo se alcanzó significación estadística cuando se comparó la tasa de SV según estadio precoz (etapa I-II) versus avanzado (III-IV), lo cual coincide con aquellos factores pronósticos que sí se asocian con una peor $\mathrm{SV}$, tales como el número de tumores, la infiltración vascular y el compromiso nodal.

\section{Señor Presidente:}

El Dr. Alexandre Sauré, cirujano digestivo, con nacionalidad chilena/italiana, nacido en la ciudad de Concepción, cursó sus estudios de pregrado en la Universidad Católica de la Santísima Concepción, egresando el año 2003; realizó su residencia de Cirugía General durante los años 2004 y 2007 en la Universidad de los Andes, con estadías en el equipo de cirugía hepatobiliopancreática y trasplante hepático del Hospital Paul Brousse de París, Francia durante el año 2006; además completó un Diplomado en "ciencias de la investigación y medicina basada en la evidencia", en la Universidad de 
Los Andes, los años 2005 y 2006. Posteriormente, realizó su formación en cirugía digestiva en el Hospital Clínico de la Universidad de Chile los años 2009 a 2011, y en la actualidad, se ha integrado al equipo de cirugía digestiva y trasplante hepático del mismo hospital.

Señor Presidente, es grato manifestar a usted, que el Dr. Sauré nos expone un trabajo interesante, bien presentado y muestra la primera experiencia de un centro nacional respecto a resultados del tratamiento quirúrgico del colangiocarcinoma intrahepático. Por los méritos señalados, sus características personales y profesionales, me permito recomendar al Dr. Sauré para que sea acogido con todas las atribuciones como Socio Titular de la Sociedad de Cirujanos de Chile.

Dr. Rodrigo Torres-Quevedo Q. (M.S.C.Ch) 